\title{
A systematic literature review of studies reporting human papillomavirus (HPV) prevalence in esophageal carcinoma over 36 years (1982-2017)
}

\author{
Lea Hošnjak ${ }^{1}$, Mario Poljak ${ }^{1 凶}$
}

\begin{abstract}
A total of 203 reports published between 1982 and 2017 on the association between human papillomaviruses (HPV) and esophageal squamous cell carcinoma (ESCC), originating from 187 studies performed in 32 countries from six continents, were selected and reviewed. The selected studies included a total of 14,788 ESCC cases; the presence of HPV infection was detected in $30.9 \%$ of cases (95\% Cl 30.1-31.6\%) and was the highest in regions with the highest incidence of ESCC. Across studies published in the last 6 years, Alphapapillomavirus species were detected in $31.1 \%$ of ESCCs $(1,464 / 4,708,95 \% \mathrm{Cl} 29.8-32.4 \%)$, of which $73.8 \%$ $(1,080 / 1,464,95 \% \mathrm{Cl} 71.5-76.0 \%)$ were positive for the presence of $\mathrm{HPV}_{16} / 18$. HPV 16 was by far the most common HPV type detected, accounting for $21.0 \%(799.5 / 3,803,95 \% \mathrm{Cl} 19.8-22.4 \%)$ of the total number of ESCC cases investigated. Our results are in line with previously published studies, suggesting the etiological role of HPV in the development of a subset of ESCC cases. Although the association between HPV and esophageal adenocarcinoma (EAC) has been studied to a far lesser extent, some studies also suggest a potential etiological role of HPV in a subset of EAC cases.
\end{abstract}

Keywords: esophageal carcinoma, human papillomaviruses, HPV, prevalence

Received: 21 July 2017 | Returned for modification: 2 August 2018| Accepted: 18 August 2018

\section{Human papillomaviruses are etiologically associated with human cancers of several anatomical sites}

Human papillomaviruses (HPVs) are a heterogeneous group of small non-enveloped viruses with a double-stranded circular DNA genome that cluster into the Papillomaviridae family. Based on the similarity of their L1 genes, HPVs are phylogenetically further classified into genera (> 60\% similarity), species (60-70\% similarity), types (> 90\% similarity), subtypes (> 98\% similarity), and viral variants (>99\% similarity of the complete genome sequence). According to the International Human Papillomavirus Reference Center, located at the Karolinska Institute in Stockholm, Sweden (http://www.nordicehealth.se/hpvcenter/reference_clones/), 221 HPV types, which cluster into five papillomavirus genera-Alphapapillomavirus (Alpha-PV), Betapapillomavirus (Beta-PV), Gammapapillomavirus (Gamma-PV), Mupapillomavirus (Mu-PV), and Nupapillomavirus ( $\mathrm{Nu}$-PV)-have been identified and officially recognized as of August 15th, 2018.

In addition to asymptomatic infections, HPVs are etiologically associated with the development of several benign and malignant lesions of the mucosal and/or cutaneous epithelium. Based on their clinical relevance, approximately 40 HPV types from the Alpha-PV genus are sub-divided into two groups: high-risk (hr) and low-risk (lr) HPVs. HrHPV types (HPV16, HPV18, HPV31, HPV33, HPV35, HPV39, HPV45, HPV51, HPV52, HPV56, HPV58, and HPV 59), considered group 1 carcinogens, are etiologically associated with more than $99 \%$ of cervical cancers, 70 to $90 \%$ of anal and vaginal cancers, $40 \%$ of vulvar cancers, $47 \%$ of penile cancers, and 25 to $30 \%$ of oropharyngeal cancers (reviewed in 1 -4). On the other hand, lrHPV types (most commonly HPV6 and HPV11) are associated with more than 90\% of anogenital warts and laryngeal papillomas (reviewed in 3). Despite the fact that Alpha-PVs cause the majority of clinically significant infections, some HPV types (HPV5, HPV8, HPV2O, HPV22, HPV 38, HPV76, and HPV92) from the Beta-PV genus can act as cofactors in the development of non-melanoma skin cancer in immunosuppressed patients (5). In addition, Gamma-, $\mathrm{Mu}$-, and $\mathrm{Nu}$-PVs are etiologically associated with a proportion of benign skin lesions (reviewed in 3).

\section{Differences in epidemiology of esophageal cancer worldwide}

According to GLOBOCAN 2012, esophageal cancer is the eighth most common cancer, with an estimated 456,000 new cases in 2012, representing $3.2 \%$ of the total number of new cancer cases worldwide (6). Moreover, esophageal cancer is the sixth most common cause of death from cancer, with an estimated 400,000 deaths in 2012, representing $4.9 \%$ of the total number of deaths caused by cancer worldwide (6).

Esophageal cancer usually develops in older patients, with a median age of 65 years, and only rarely in individuals under 30 (reviewed in 7 and 8). Worldwide, the incidence of esophageal cancer varies between 0.8 and 17.0 per 100,000 in males and 0.2 and 7.8 per 100,000 in females, suggesting that esophageal cancer is a male-predominant disease $(323,000$, or $70.8 \%$ of new cancer cases in 2012) (6). In addition, in low-income countries, males are at a twice greater lifetime risk of developing esophageal cancer in comparison to women, and the difference can be up to four times greater in some high-income countries (6).

In comparison to more developed regions, the incidence of esophageal cancer is more than four times higher in less-developed regions (6). Furthermore, up to 500-fold differences have been observed across different countries/areas and even between different ethnic groups within the same area (9). As shown in Table 1 , in 2012, the estimated overall age-adjusted incidence rates 
standardized for the world population (ASR(w)s) per 100,000 were the highest in East Africa (Kenya) (17.6), China (12.5), South Africa (9.9), and Iran (8.6) (6), confirming the results of previous studies, which suggested that high-risk areas for esophageal cancer stretch from East Asia to Central Asia (the so-called esophageal cancer belt) and along the Rift Valley in East Africa into South Africa, with incidence rates as high as 246 per 100,000 (reviewed in 7 and 10). In 2012, 3,432 new esophageal cancer cases were diagnosed in both genders in Kenya. In the same year, 223,306 new esophageal cancer cases were recorded in China alone, confirming that China is still the most affected country worldwide (reviewed in 7), with esophageal cancer being the fourth most common cause of death from cancer, accounting for 9.8 and $7.4 \%$ of all cancer deaths among men and women, respectively (6). Interestingly, in comparison to urban areas (e.g., Beijing and Shanghai), the incidence of esophageal cancer is higher in rural areas of China (the highest in Henan province) (reviewed in 7). According to GLOBOCAN 2012, 3,871 and 5,343 new esophageal cancer cases were diagnosed in both genders in South Africa and Iran, respectively (6). Similar to in China, some parts of Iran (especially Turkmen Sahra) also have a higher incidence of esophageal cancer (reviewed in 7). As shown in Table 1, the population in high-income countries is at lower risk for development of esophageal cancer, with ASR(w)s per 100,000 of 3.5, 3.3, and 3.1 in Australia, Europe, and North America, respectively (reviewed in 6 and 7).

Although several histological subtypes of esophageal cancer have been described to date, esophageal squamous cell carcinoma (ESCC), arising from squamous epithelial cells, and esophageal adenocarcinoma (EAC), arising from glandular epithelial cells, are the most common, together representing more than $90 \%$ of all esophageal cancer cases. Whereas ESCC predominates in the upper and middle esophagus, EAC usually arises in the lower esophagus, near the gastric junction (reviewed in 11 and 12). The first study that evaluated the global burden of ESCC and EAC, respectively, reported that an estimated 398,000 new cases of ESCC and 52,000 new cases of EAC were diagnosed in 2012 (13). The male-to-female ratio of the probability of developing esophageal carcinoma was 4.4 and 2.7 for EAC and ESCC, respectively (13). Whereas the majority (79\%) of ESCC cases occurred in less-developed regions (Southeast and Central Asia), the incidence of EAC was the highest in more developed countries (northern and western Europe, North America, and Oceania), where $46 \%$ of all EAC cases have been diagnosed (13). Interestingly, even though ESSC is still the predominant esophageal cancer subtype worldwide, in several high-income countries (Australia, Bahrain, Canada, Cyprus, Iceland, Ireland, Malta, the Netherlands, New Zealand,

Table 1 | HPV DNA prevalence in esophageal squamous cell carcinoma according to geographic region and country of origin (1982-2017).

\begin{tabular}{|c|c|c|c|c|c|c|c|}
\hline Geographic region & $\begin{array}{c}\text { Incidence of ESCC } \\
\text { ASR }(w)^{a}\end{array}$ & Risk of ESCC ${ }^{b}$ & $\begin{array}{l}\text { No. of samples } \\
\text { tested }\end{array}$ & $\begin{array}{c}\text { No. of HPV-positive } \\
\text { samples }\end{array}$ & $\begin{array}{c}\text { HPV prevalence } \\
(\%)\end{array}$ & $\begin{array}{l}\text { Range of HPV } \\
\text { positivity (\%) }\end{array}$ & $\begin{array}{c}\text { No. of } \\
\text { reports }^{c}\end{array}$ \\
\hline East Africa (Kenya) & 17.6 & high & 29 & 0 & 0 & & 1 \\
\hline China & 12.5 & high & 8,503 & 3,404 & 40.0 & $0-100$ & 73 \\
\hline South Africa & 9.7 & high & 641 & 165 & 25.7 & $2.2-66.7$ & 13 \\
\hline - South Africa & 9.9 & high & 549 & 163 & 29.7 & $8.8-66.7$ & 12 \\
\hline - Zambia & 9.1 & high & 92 & 2 & 2.2 & & 1 \\
\hline Other Asia & $\mathrm{N} / \mathrm{A}$ & high & 2,527 & 536 & 21.2 & $0-100$ & 44 \\
\hline - India & 4.1 & high & 349 & 107 & 30.7 & $5.0-100$ & 7 \\
\hline - Iran & 8.6 & high & 937 & 208 & 22.2 & $0-49.4$ & 13 \\
\hline - Japan & 6.1 & high & 974 & 176 & 18.1 & $0-63.0$ & 19 \\
\hline - Korea & 2.9 & low-medium & 225 & 34 & 15.1 & $0-66.7$ & 4 \\
\hline - Pakistan & 4.1 & $\mathrm{~N} / \mathrm{A}$ & 42 & 11 & 26.2 & & 1 \\
\hline Latin America & 4.3 & low-medium & 816 & 136 & 16.7 & $0-100$ & 13 \\
\hline - Brazil & 6.1 & low-medium & 609 & 74 & 12.2 & $0-15.8$ & 6 \\
\hline - Chile & 3.2 & low-medium & 26 & 5 & 19.2 & & 1 \\
\hline - Colombia & 1.9 & low-medium & 102 & 25 & 24.5 & $0-34.0$ & 3 \\
\hline - Mexico & 1.0 & low-medium & 77 & 30 & 39.0 & $25.0-88.2$ & 2 \\
\hline - Venezuela & 1.2 & low-medium & 2 & 2 & 100 & & 1 \\
\hline Australia & 3.5 & low-medium & 370 & 23 & 6.2 & $1.0-50.0$ & 4 \\
\hline Europe & 3.3 & low-medium & 1,347 & 197 & 14.6 & $0-100$ & 35 \\
\hline - Belgium & 4.6 & low-medium & 23 & 3 & 13.0 & $4.8-100$ & 3 \\
\hline - Bulgaria & 1.6 & low-medium & 4 & 1 & 25.0 & & 1 \\
\hline - Finland & 2.3 & low-medium & 121 & 35 & 28.9 & $18.0-40.0$ & 2 \\
\hline - France & 3.8 & low-medium & 171 & 6 & 3.5 & $0-41.7$ & 5 \\
\hline - Germany & 4.0 & low-medium & 76 & 9 & 11.8 & $0-17.0$ & 2 \\
\hline - Greece & 0.8 & low-medium & 49 & 19 & 38.8 & $10.5-56.7$ & 2 \\
\hline - Hungary & 3.6 & low-medium & 26 & 6 & 23.1 & & 1 \\
\hline - Italy & 1.3 & low-medium & 136 & 20 & 14.7 & $0-47.1$ & 5 \\
\hline - Netherlands & 6.3 & low-medium & 124 & 0 & 0 & & 2 \\
\hline - Poland & 2.2 & low-medium & 56 & 28 & 50.0 & & 1 \\
\hline - Portugal & 3.1 & low-medium & 16 & 9 & 56.3 & & 1 \\
\hline - Slovenia & 2.1 & low-medium & 141 & 2 & 1.4 & $0-10.0$ & 2 \\
\hline - Sweden & 2.3 & low-medium & 314 & 36 & 11.5 & $0-16.0$ & 3 \\
\hline - Turkey & 3.6 & low-medium & 63 & 22 & 34.9 & $9.1-63.3$ & 2 \\
\hline - United Kingdom & 6.5 & low-medium & 27 & 1 & 3.7 & $0-100$ & 3 \\
\hline North America & 3.1 & low-medium & 369 & 79 & 21.4 & $0-100$ & 19 \\
\hline North Africa (Egypt) & 2.1 & low-medium & 50 & 27 & 54.0 & & 1 \\
\hline $\begin{array}{l}\text { Total according to } \\
\text { geographic region }\end{array}$ & & & 14,788 & 4,567 & 30.9 & $0-100$ & 203 \\
\hline
\end{tabular}

ancidence of ESCC in ASR (w): age-standardized incidence rate per 100,000 (6).

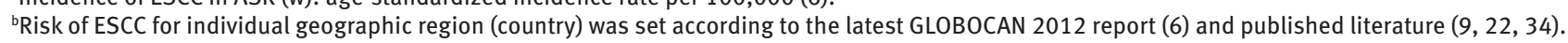

'Total number of reports extracted from 187 studies included in the analyses. ASR(w) = age-standardized incidence rate standardized for the world population per 100,$000 ; E S C C=$ esophageal squamous cell carcinoma; N/A = not available. 
Norway, Sweden, the United Kingdom, and the United States) the incidence of EAC has already exceed that of ESCC (13). Moreover, in the next 10 years, the burden of EAC is expected to increase rapidly, resulting in EAC being the predominant subtype of esophageal cancer at least in some high-income countries (14). Nevertheless, esophageal carcinoma will remain an important healthcare problem in less-developed regions, where 369,640 new cancer cases were diagnosed in 2012, in comparison to 86,144 in more developed regions (6).

In addition to epidemiological differences between ESCC and EAC, a recent study identified several molecular features that support the current histological differentiation of esophageal carcinoma (15). Based on the comprehensive molecular analysis of 164 esophageal carcinomas, Kim et al. proposed that ESCC is more similar to squamous cell carcinoma of the head and neck region than EAC, whereas EAC resembles a chromosomally unstable variant of gastric adenocarcinoma (15).

\section{Unresolved etiology of esophageal cancer}

As described above, esophageal cancer includes several different histological subtypes of cancer (reviewed in 11 and 12). In addition, inhabitants of low-income countries have a higher risk of developing esophageal cancer, especially ESCC, in comparison to those living in high-income countries (reviewed in 6 and 13). Thus the etiology of esophageal cancer is most likely multifactorial and is still unresolved (reviewed in 7). The most common risk factors proposed for EAC include obesity, tobacco smoking, gastroesophageal reflux disease, Barrett's esophagus, and a diet low in fruits and vegetables. Interestingly, the etiology of ESCC differs substantially between low- and high-endemic areas; ESCC in low-endemic areas is mostly caused by chronic cell damage as a consequence of tobacco smoking and high alcohol consumption, especially both risk factors together. On the other hand, in high-endemic areas, ESCC most probably develops as a consequence of synergistic effects of chemical risk factors (nitrosamines and their precursors, environmental pollutants, opium, alcohol, tobacco, processed red meat, and nutritional deficiency), physical risk factors (thermal damage to the esophageal mucosa, achalasia, and radiation), and biological risk factors (bacteria and their metabolites, which increase cellular proliferation and stimulate the inflammatory process; fungi, which produce mycotoxins with tumorigenic properties; and some viruses) (reviewed in 8, 7, 11 and 12). In addition, based on the results of three genome-wide association studies, which described several new susceptibility loci, it has been suggested that ESCC could also be attributed to some genetic factors (16).

Since the first report proposing an etiological role of HPV in the development of neoplasms of the esophagus, published in 1982 (17), the arguments for etiological association between HPV and a subset of ESCC cases are becoming stronger with time, with several studies published in the last 36 years.

\section{Thirty-six years of confusing results on the etiological association between HPV and ESCC}

In the last 36 years, over 200 geographically diverse peer-reviewed publications, spanning from case reports to case-control studies, have reported the prevalence of HPVs in patients with ESCC. Nevertheless, when considering each study separately, the results are inconclusive because the reported HPV prevalence in ESCC varies from to o to $100 \%$. Interestingly, HPV was not even mentioned as a possible etiological agent in the development of a subset of ESCCs in two recent review articles published in The Lancet $(11,12)$, nor is the role of hrHPVs in association with ESCC recognized by the International Agency for Research on Cancer, in contrast to the role of HPVs in the development of various anogenital and oropharyngeal cancers of both genders (reviewed in 7). We therefore believe that periodical systematic reviews and meta-analyses can be of great help when critically evaluating and building knowledge concerning the potential role of hrHPV in the development of ESCC.

\section{Summary of main systematic reviews and meta-analyses on the role of HPV in the development of ESCC}

Following the first formal literature review of the role of HPV in the development of ESCC, which was published by our group in 1998 (18), Kari Syrjänen published several updated literature reviews in 2002, 2006, 2010, and 2013 (19-22) followed by others. Briefly, in the last systematic review and meta-analysis published by Syrjänen in 2013, 152 studies dating from 1982 to March 2012 and originating from 32 different countries were selected and analyzed. Out of 10,234 ESCC cases analyzed, 3,135 (30.6\%) were HPV positive. However, the HPV prevalence varied greatly across different geographic regions, with the highest HPV prevalence recorded in regions with the highest incidence of ESCC: China $(2,268 / 5,651 ; 40.1 \%)$, South Africa $(176 / 468 ; 37.6 \%)$, and Latin America (80/357; 22.4\%). Similarly, HPV prevalence was the lowest in ESCC low-incidence regions: North America: 8.0\% (18/226), Australia: 8.1\% (22/271), and Europe: $14.0 \%$ (132/944). The results suggested that the etiology of ESCC differs between low- and highincidence regions, with HPV playing an important role only in high-incidence regions (22).

In 2014, our research group published an updated systematic review on the role of HPVs in the development of ESCC, which included 159 studies published between 1982 and August 2013, originating from 33 countries from five continents (7), using a slightly different approach from that of Syrjänen in 2013 (22). In our literature review, a total of 11,310 ESCC cases tested for the presence of HPV were analyzed and the overall prevalence of HPV infection was estimated at 30.3\%, with important geographical differences, similar to what was obtained in Syrjänen's 2013 review (22). In addition, 42 studies, published between 2008 and 2013, including 4,014 ESCC patients from 17 countries worldwide, were eligible and critically assessed for type-specific HPV prevalence data. In these 42 studies, the overall Alpha-PV prevalence was $32.2 \%$ $(1,291 / 4,014)$. The calculated combined HPV16/18 prevalence was $23.3 \%$, with HPV16 and HPV18 found in $16.3 \%$ and $2.7 \%$ of ESCCs investigated, respectively. Moreover, the combined HPV16/18 prevalence among all HPV-positive cases was estimated at 73.7\% (7).

In 2013, The Australian research group subsequently published a first global meta-analysis of case-control studies investigating the association between HPV and ESCC (9). A total of 21 case-control studies, dating from 1991 to 2010, including 1,223 and 1,415 patients with ESCC and control subjects, respectively, were analyzed in the meta-analysis. In contrast to $35 \%$ of Alpha-PV-positive ESCC cases, HPV DNA was detected in $27 \%$ of control cases, irrespective of the HPV detection method used: immunohistochemistry (IHC), in-situ hybridization (ISH), and/or polymerase chain reaction (PCR). Furthermore, this meta-analysis provided strong evidence of an etiological association between HPV and ESCC, with a calculated pooled odds ratio (OR) of 3.04 ( $95 \%$ confidence interval 
(CI) 2.2-4.2). Interestingly, in comparison to countries with a high incidence of ESCC, the association was stronger in countries with a low to medium incidence of ESCC (OR 2.65, 95\% CI 1.80-3.91 vs. OR 4.65, 95\% CI 2.47-8.76) (9).

The geographical differences in HPV prevalence among ESCC cases observed in previous studies $(7,9,22)$ were subsequently also confirmed in the meta-analysis by Liu et al., which critically addressed a total of 145 ESCC studies published between 1982 and 2012 (23). Among 6,912 ESCC cases, the HPV prevalence was estimated at $38.9 \%$ and was statistically significantly higher in China $(44.0 \%)$ in comparison to other regions $(31.2 \%)(p<0.05)$. In addition, similar to Liyanage et al. (9), the meta-analysis by Liu et al. confirmed that ESCC is etiologically associated with HPV infection (OR 4.20, 95\% CI 3.08-5.74) (23).

A comprehensive meta-analysis by Hardefeldt et al. selected a total of 132 studies published from 1986 to 2012 (24). The overall prevalence of HPV in ESCC cases was 24.8\% (2,985/12,037, 95\% CI 21.2-28.8\%). Similar to previous studies (9, 23), Hardefeldt et al. found evidence of an increased risk of ESCC development in patients with HPV-positive cancers (OR 2.69, 95\% CI 2.05-3.54) and also an increased risk associated with HPV16 infection (OR 2.35, 95\% CI 1.73-3.19). Moreover, the meta-analysis confirmed geographical differences in the risk of ESCC development, with Asia (OR 2.94, 95\% CI 2.16-4.00), and especially China (OR 2.85, 95\% CI 2.05-3.96), characterized as high-risk regions (24).

In 2014, Li et al. published a systematic review with a metaanalysis that assessed 8,990 ESCC cases obtained from 76 studies (25). In addition to the overall HPV prevalence in ESCCs, which was $22.2 \%$ (95\% CI $18.3-26.7 \%$ ), the study also provided a typespecific HPV prevalence, with HPV16 being the most frequently detected HPV type, with an overall prevalence of $11.4 \%$ (95\% CI 8.2-15.7\%), similar to what was observed in previous studies (7, 24). In line with the previous studies (9, 23, 24), Li et al. also observed a significant association between ESCC and HPV infection (OR 3.32, 95\% CI 2.26-4.87) and ESCC and HPV16 infection (OR 3.52, 95\% CI 2.04-6.07) (25).

In 2013, Yong et al. published a meta-analysis of the etiological role of HPV16 and HPV18 in the development of ESCC, in which they analyzed 68 studies published between 1989 and September 2012 (26). The overall HPV16 and HPV18 positivity rates in ESCC cases were $11.7 \%$ (95\% CI 7.74-16.21\%) and 1.8\% (95\% CI 0.90-2.95\%), respectively. In addition, a meta-analysis of 10 case-control studies, including 1,130 ESCC cases and 1,614 controls, revealed a significantly increased risk of ESCC in patients with HPV16 infection (OR 3.55, 95\% CI 2.0-6.14), similar to previously published studies $(24,25)$. In contrast, based on an analysis of six case-control studies, including 750 ESCC cases and 1,356 controls, they showed that HPV18 infection is not associated with an increased risk of ESCC development (OR 1.25, 95\% CI 0.46-3.43) (26).

Zhang et al. conducted a systematic review and a meta-analysis of HPV16 prevalence among Chinese patients with ESCC, including studies published from 2005 to July 2014 (27). A total of 3,429 ESCC cases, originating from 26 Chinese studies, were included in the meta-analysis. Although the estimated overall prevalence of HPV16 infection was 38.1\% (95\% CI 28.3-47.9\%), significant differences were observed according to the geographical region, publication year, types of specimens tested, and HPV detection method used. Specifically, the HPV16 prevalence varied from $39.7 \%$ (95\% CI 24.9-54.5\%) in the northern part of China to $47.8 \%$ (95\% CI 36.2-59.4\%) in the southern part of the country. In comparison to studies published between 2010 and 2014, the
HPV16 prevalence was higher in studies that were published between 2005 and 2009 (36.2\%, 95\% CI $22.3-50.1 \%$ vs. $40.2 \%$, 95\% CI $0.238-0.565 \%$ ). As expected, the observed HPV16 prevalence was higher in studies conducted on fresh-frozen tissue samples (43.3\%, 95\% CI 23.5-63.1\%) than on paraffin-embedded tissues (36.5\%, 95\% CI 24.2-48.9\%). Interestingly, the HPV16 prevalence also differed if HPV detection methods targeted different parts of the viral genome, with higher HPV16 prevalence observed when targeting E6/E7 genes in comparison to the L1 gene (47.4\%, 95\% CI $37.3-57.4 \%$ vs. $28.8 \%$, 95\% CI 18.0-39.6\%) (27). Subsequently, the same research group conducted a meta-analysis of 10 casecontrol studies, selected from the original set of studies, together including 1,442 ESCC patients and 1,602 control subjects (28). With ORs ranging from 3.65 (95\% CI 2.17-6.13) to 15.44 (95\% CI 3.42-69.70) and a pooled estimate of 6.36 (95\% CI 4.46-9.07), HPV16 was identified as a risk factor for the development of esophageal cancer in China (28). Furthermore, the same research group published a similar study on HPV18, including 19 studies with a total of 2,556 ESCC patients (29). The pooled HPV18 prevalence in ESCC cases was estimated at 4.1\% (95\% CI 2.7-5.5\%) and ranged from $0 \%$ to $26.1 \%$; however, the infection with HPV18 was not associated with an increased risk for ESCC development in China (29), confirming the results of the global analysis (26).

Michaelsen et al. conducted a systematic review of studies published between 1980 and July 2013 to assess whether overexpression of $\mathrm{p}_{16} 6^{\mathrm{INK} 4 \mathrm{~A}}$ can be used as a marker of transcriptionally active HPV in ESCCs. A total of 12 studies, including 1,383 ESCC cases, originating from 10 different countries, were included in the systematic review. HPV DNA were detected in 12\% $(161 / 1,347)$ of cases and $33.9 \%(209 / 617)$ of ESCC cases were $\mathrm{p} 16^{\mathrm{INK} 4 \mathrm{~A}}$ positive, suggesting that the two markers are unrelated (30). The results of this study were further confirmed by Wang et al. (31), who showed that $16^{\mathrm{INK} 4 \mathrm{~A}}$-positive expression was associated with lymph node metastasis and that p53-negative expression may be used as a reliable marker for HPV status in ESCC (31), and by Halec et al., who performed a meta-analysis of nine case-control studies, published up to December 2014, and additionally tested 116 ESCC tissue samples (32).

The systematic review with the largest number of ESCCs included so far-13,832 samples originating from 124 studies published up to July 2013-was conducted by Petrick et al. (33). Interestingly, the HPV prevalence rate primarily depended on the HPV detection method used, being the highest using L1 serology (32.2\%, 95\% CI 15.4-49.0\%), followed by IHC (30.4\%, 95\% CI 18.5-42.3\%), PCR (27.7\%, 95\% CI 23.4-32.0\%), ISH (24.3\%, 95\% CI 15.9-32.6\%), and Southern/slot/dot blot (17.6\%, 95\% CI 6.1-29.2\%). In accordance with previous studies $(7,9,22-24,34)$, the detection rate of HPV varied across different geographical regions, with the HPV prevalence ranging from $15.6 \%$ (95\% CI 7.3-23.8\%) in Europe/Australia to $31.4 \%$ (95\% CI $27.4-35.4 \%$ ) in Asia. Differences in HPV prevalence were also observed between studies that were published prior to 1990 (10.3\%, 95\% CI 11.4-32.1\%), between 1990 and 1999 (20.4\%, 95\% CI 14.9-25.9\%), and after 2000 (31.0\%, 95\% CI 26.7$35.2 \%$ ), and between studies including fewer than 60 ESCC cases (27.2\%, 95\% CI 22.6-31.8\%) and studies with more than 6o ESCC cases $(26.1 \%$, 95\% CI 21.1-31.1\%). Similar to previous literature reviews $(7,24-26,28)$, HPV16 was the predominant HPV type, with an estimated prevalence of $18.5 \%$ (95\% CI 14.2-22.8\%), when considering studies using PCR as an HPV-detection method (33).

The largest meta-analysis of case-control studies, which included 33 reports dating from between 1982 and 2014, with a total 
of 2,430 ESCC patients and 3,621 control subjects, was published by Wang et al. (35). In agreement with Liyanage et al. (9), they similarly observed a higher HPV infection rate in the ESCC group (46.5\%) than in the control group (26.2\%) (OR 1.62; 95\% CI 1.331.98). In addition, they also reported significant differences in the strength of association between HPV infection and ESCC due to use of different HPV detection methods, with the highest OR observed in studies based on IHC (1.69, 95\% CI 0.96-2.96), followed by PCR (1.61, 95\% CI 1.33-1.95), serology (1.28, 95\% CI 0.54-3.04), and ISH (1.21, 95\% CI 0.62-2.36) (35). Because the reported values in the largest meta-analysis of case-control studies differs from those observed previously by Liyanage et al. and Petrick et al. (9, 33), further studies are needed to clarify whether different HPV methodologies are confounding factors in studies investigating the etiological association between HPV and ESCC.

Taken together, the results of previously published systematic reviews and meta-analyses suggest that hrHPVs, particularly HPV16, could play an etiological role in the development of a subset of ESCC cases. However, due to substantial differences in the geographical distribution of (HPV-positive) ESCC cases and suggested differences in HPV detection rates due to study sizes, types of specimens tested, and HPV detection methods used, further studies are warranted.

To the best of our knowledge, no systematic reviews and/ or meta-analyses, including a plethora of studies published between January 2015 and December 2017, are available in the peerreviewed literature. As described above, our research group published the last systematic literature review on the topic in 2014, in which we assessed 159 studies published between 1982 and August 2013 (7). Here we provide an updated systematic literature review covering all eligible studies that were published until the end of 2017, with special emphasis on the most recent data.

\section{An updated systematic literature review of studies reporting HPV prevalence in ESCC (1982-2017)}

In order to identify eligible peer-reviewed studies, several webbased databases, including Medline/PubMed, Web of Science, Scopus, and Google Scholar, were searched for a combination of the following terms: papillomavirus, human papillomavirus, HPV, epidemiology, esophagus, oesophagus, cancer, and carcinoma. Following an initial search, which was performed on August 29th, 2017, the search was repeated on December 28th, 2017, and subsequently additional studies were singled out through the revision of reference lists of previously identified studies. To be eligible for this review, studies needed to fulfill the following criteria: i) a defined population of patients, ii) at least one patient diagnosed with ESCC, iii) well-described HPV detection method(s), iv) numbers of HPV-tested and HPV-positive samples provided, and v) results not published in duplicate/multiple publications. Date of publication, publication language, and choice of the HPV detection method were not limiting factors.

As shown in Table 1, a total of 203 reports originating from 187 studies and 32 countries from six continents (Africa, Asia, Australia, Europe, North America, and Latin America) published between 1982 and 2017 were selected and analyzed. Since our last systematic literature review article (7), 28 additional original and eligible studies have been published, with the majority of new studies originating from China (16/28; 57.1\%) (Table 2).

The overall HPV prevalence among 14,788 ESCC cases was $30.9 \%$ (95\% CI 30.1-31.6\%) (Table 1), in the range that was as- sessed in previously published systematic reviews and meta-analyses $(7,9,22)$. Although HPV prevalence ranged from o to $100 \%$ in the majority of regions, it was overall the highest in regions with the highest incidence of ESCC: China (40.0\%, 95\% CI 39.0-41.1\%), South Africa (29.7\%, 95\% CI 26.0-33.6\%), and several Asian countries, except China (21.2\%, 95\% CI 19.7-22.9\%), confirming the results of previous studies $(7,22-24,33)$. Among Asian countries, the overall HPV prevalence was the highest in India, Pakistan, and Iran (Table 1). Interestingly, no HPV-positive cases were observed in the single study originating from East Africa (Kenya), with the highest estimated worldwide incidence of ESCC in 2012 (6). In low to medium ESCC risk regions, HPV prevalence was the highest in North Africa (Egypt) (27/50, 54.0\%, 95\% CI 40.4-67.0\%), followed by North America (79/369, 21.4\%, 95\% CI 17.5-25.9\%), Latin America (136/816, 16.7\%, 95\% CI 14.3-19.4\%), Europe (197/1,347, 14.6\%, 95\% CI 12.8-16.6\%), and Australia (23/370, 6.2\%, 95\% CI 4.2-9.2\%). In line with our previous literature review (7), in Latin American and European countries no association was observed between estimated HPV prevalence and the risk of ESCC (Table 1).

\section{A review of type-specific prevalence in ESCC cases over the last 6 years}

In the last 6 years, a total of 42 publications, originating from 12 different countries (Australia, Brazil, China, Greece, India, Iran, Poland, South Africa, Sweden, Turkey, the United States, and Zambia) from six continents, reported the type-specific HPV prevalence in ESCCs (Table 2). As shown in Table 2, in the vast majority of studies, HPV typing was performed by PCR-based methods, either using commercial Alpha-PV DNA-based tests or in-house consensus and type-specific PCRs. In two studies novel broader and more sensitive HPV typing methods were used: matrix-assisted laser desorption/ionization time-of-flight mass spectrometer (MALDI-TOF MS) and Luminex technology (Luminex, Austin, TX, US) (45, 67). Older, less sensitive, and less specific HPV typing methods such as IHC and ISH were used in only one and five studies reviewed, respectively $(40,48,72-74,75)$, suggesting the predominance of more sensitive and specific PCR-based methods in the most recent studies.

In studies published in the last 6 years, the overall Alpha-PV prevalence was 31.1\% (1,464/4,708, 95\% CI 29.8-32.4\%), ranging from o to $100 \%$ in individual studies (Table 2), similar to what was recorded in previous systematic reviews $(7,9,22,33,34)$. In addition, in line with previously published reviews (7, 24-29, 33), the combined HPV16/18 prevalence among Alpha-PV-positive ESCCs was $73.8 \%$ (1,080/1,464, 95\% CI 71.5-76.0\%; Table 2), suggesting that any of the three currently approved prophylactic HPV vaccines have the potential to prevent more than two-thirds of all HPV-positive ESCCs. As shown in Table 2, the calculated combined general HPV16/18 prevalence among all ESCCs was $26.3 \%$ (1,080/4,113, 95\% CI 25.0-27.6\%), with HPV16 being by far the most common HPV type, accounting for $21.0 \%$ (799.5/3,803, 95\% CI 19.8-22.4\%) of all ESCCs tested, followed by HPV18 present in $5.5 \%$ of ESCCs $(122.5 / 2,226$, 95\% CI 4.7-6.6\%). Our results are in line with previously published studies $(24-26,28,33,36)$. In addition, several other HPV types (HPV6, HPV11, HPV31, HPV32, HPV33, HPV35, HPV39, HPV42, HPV43, HPV45, HPV51, HPV52, HPV56, HPV58, HPV59, HPV66, HPV70, and HPV82) from the Alpha-PV genus were detected in ESCC tissue samples, mostly as coinfections with HPV16 and HPV18 (Table 2), similar to what was observed in our last literature review (reviewed in 7). 


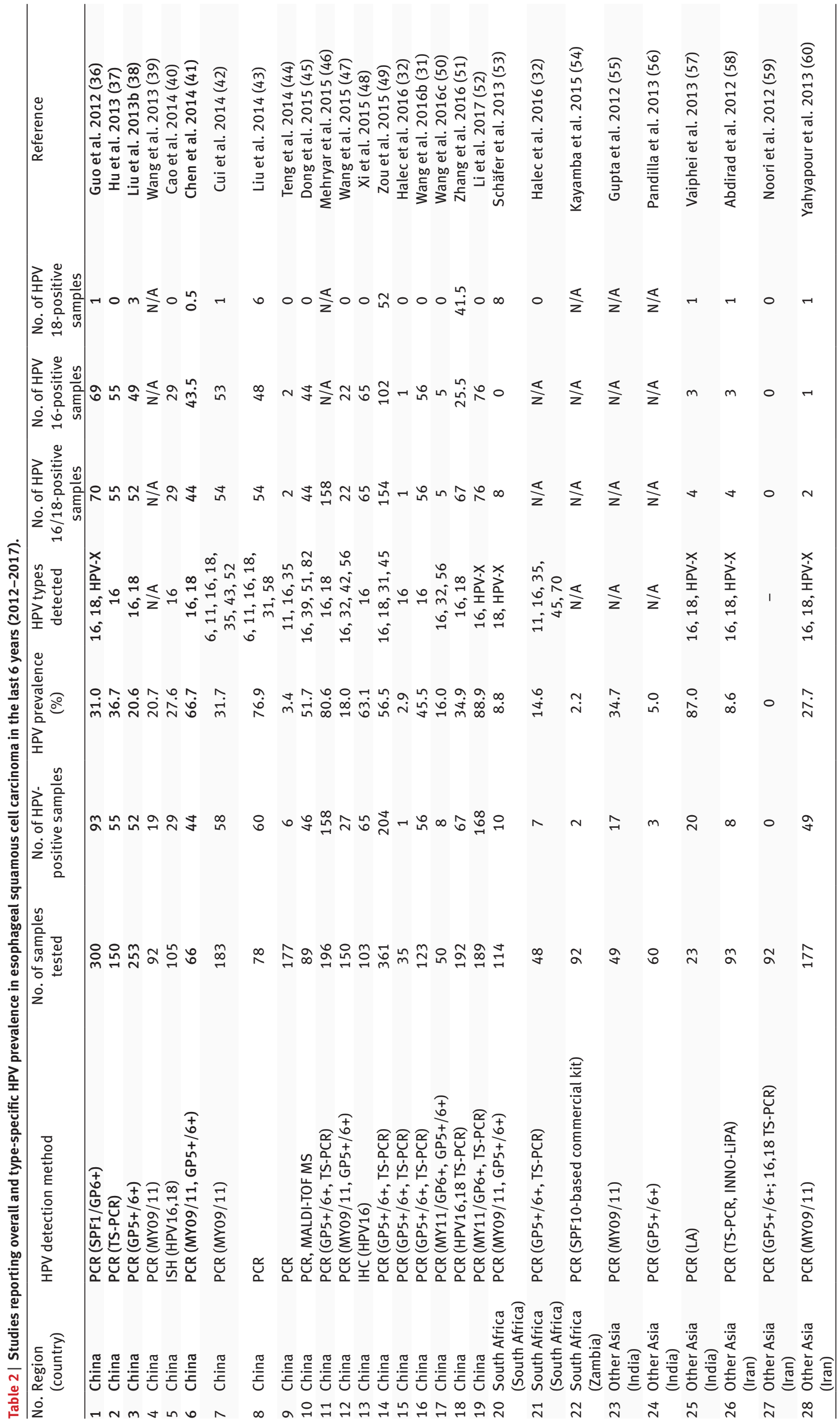









\section{HPV infection is associated with prognosis of patients with ESCC}

In a recent case-control study, which included 103 ESCC cases and 54 controls obtained from Chinese patients between September 2008 and December 2009, Xi et al. (48) confirmed the results of previous studies showing a high prevalence of HPV infection among ESCC patients in China (7, 22-24, 27, 33). Moreover, they showed that HPV16 infection and high phosphatidylinositol 3-kinase ( $\left.\mathrm{PI}_{3} \mathrm{~K}\right)$ expression levels are independently negatively correlated with 3-year and 5-year overall survival and progressionfree survival and can therefore be used as markers for predicting disease outcome in patients with ESCC (48). The same research group performed a subsequent study investigating the HPV16-mediated mechanisms linked with the development and progression of ESCC and showed that HPV16 E6 and E7 proteins can promote cancer stem-like cells' phenotype in ESCC cells by activating the $\mathrm{PI}_{3} \mathrm{~K} /$ Akt signaling pathway, which can potentially be targeted in treatment of ESCC (76).

\section{The role of HPV in the development of EAC}

In addition to obesity, smoking, gastroesophageal reflux disease, Barrett's esophagus, and a diet low in fruits and vegetables, traditionally described as the risk factors for the development of EAC (reviewed in 11 and 12), some research groups report that hrHPV infection could also be involved in the etiopathogenesis of a subset of EAC cases (reviewed in 77 and 78). The cumulative overall HPV prevalence obtained in six studies published between 2000 and 2016, including a total of 404 EAC cases, was $7.7 \%$ (31/404, 95\% CI 5.5-10.7\%) (79-85). Rajendra et al. (Rajendra et al. 2013) performed a case-control study, including 261 patients with Barrett's esophagus, Barrett's dysplasia, and EAC and 51 healthy controls. In comparison to the control group, the HPV prevalence was significantly higher in Barrett's dysplasia (68.6\%, incidence rate ratio (IRR) $2.94,95 \% \mathrm{CI} 1.78-4.85, p<0.001)$ and EAC $(66.7 \%$, IRR 2.87, 95\% CI 1.69-4.86, $p<0.001)$. In addition, 75/81 (92.6\%) HPV-positive patients were infected with hrHPVs (HPV16, HPV18). Moreover, when testing for HPV E6/E7 mRNA expression using a nested reverse transcription PCR, expression of mRNA for these two most important HPV oncogenes was demonstrated in 9/22 $(40.9 \%)$ and $9 / 15(60.0 \%)$ of Barrett's dysplasia and EAC cases, respectively, whereas all controls and patients with Barrett's esophagus were without measurable oncogene expression $(p<0.001)$. Furthermore, Rajendra et al. showed a strong association between disease severity, the presence of HPV DNA, and expression of E6/ E7 mRNA and p16 ${ }^{\text {INK4A }}$ (OR 62.2, 95\% CI 12.4-311, $p<0.001$ ) (85). The same research group subsequently performed a whole exome sequencing analysis of HPV-positive and HPV-negative EAC cases and revealed the genomic differences between both groups (86). Namely, HPV-positive cases harbored 50\% less non-silent somatic mutations in comparison to HPV-negative cases (1.31 mutations/ $\mathrm{Mb}$ vs. 2.56 mutations/Mb, $p=0.048$ ), similar to what was observed previously in other head and neck cancers as well as cervical cancer $(87,88)$. In addition, Rajendra et al. confirmed the results of their previous study (89), showing the absence of aberrations in the gene encoding the tumor suppressor protein p53 (wild-type) among HPV-positive cases, in contrast to HPV-negative tumors, which frequently (up to 50\%) contained p53 mutations (86). In their most recent study, Rajendra et al. complemented their previous results and concluded that the active role of HPV in the development of Barrett's dysplasia and EAC can be indirectly determined by detection of wild-type p 53 and aberrations of the retinoblastoma protein (pRB) pathway (90).

\section{Conclusion}

Esophageal cancer is the eighth most common cancer and sixth most common cause of death from cancer worldwide (6). The etiology of esophageal cancer is most likely multifactorial and is still unresolved. Several studies performed in the last 36 years have suggested HPV as a potential risk factor for the development of the two most frequent histological subtypes of esophageal carcinoma: ESCC and EAC.

To the best of our knowledge, this literature review covers the most complete list of studies on the association between HPV and the development of esophageal carcinoma published in peer-reviewed journals between 1982 and 2017. The overall HPV prevalence identified in our review was $30.9 \%$ and $7.7 \%$ among 14,788 ESCC and 404 EAC cases, respectively. Infections with HPV16 and HPV18 were the most common, suggesting than more than two-thirds of all HPV-positive esophageal carcinomas could theoretically be prevented using any of the three current prophylactic HPV vaccines, although only if proved to also work against nongenital HPV-related cancers or their precursors.

In order to provide conclusive evidence that hrHPVs are definitive causative factors of ESCC, we need more studies. In our opinion, a well-designed large case-control study with sufficient power to indisputably ascertain HPV rates in ESCC cases compared with controls without ESCC is the most practical way forward (7). The proposed large case-control study should preferably be an international collaboration bringing together the best researchers from all continents, and should use a mandatory uniform HPV DNA (and possibly HPV RNA) testing methodology for all tissue samples. In a perfect scenario, all tissue samples should be processed (e.g., cut from paraffin blocks) and tested for HPV DNA in a single central laboratory with long experience in dealing with archival clinical specimens (7). ESCC tissue samples and tissue samples of normal esophageal mucosa should be collected from countries/areas covering the entire spectrum of ESCC incidence rates, from extremely low-incidence areas to areas with the highest incidence rates of ESCC, with minimal variations in specimen retrieval and storage. The study should not use tissue adjacent to the ESCC lesion (histologically normal esophageal tissue from resection specimens of ESCC) as "controls" because there is a high potential for cross-contamination and spread of HPV from tumor tissue to adjacent non-malignant esophageal tissue, creating false-positive results in the detection of HPV DNA in the nontumor tissue $(7,91)$. Finally, the case-control study should collect data on potential confounders and effect modifiers, and these should be adjusted for when examining the effect of HPV. These include at least age, gender, smoking, alcohol consumption, family history of esophageal cancer, pre-existing immunosuppression prior to cancer diagnosis, a history of thoracic irradiation, socioeconomic status, diets high in processed red meat, consumption of hot food and beverages, pickled foods, and diets low in fresh fruit and vegetables (7).

In conclusion, in order to provide conclusive evidence that HPV is a definitive causative factor of a subset of ESCC, any future case-control study to address the HPV-ESCC relationship would need to be substantially larger and planned in a substantially different way than any of the currently published studies. 


\section{Funding}

The authors would like to acknowledge financial support from the Institute of Microbiology and Immunology, Faculty of Medicine, University of Ljubljana, the Slovenian Research Agency (research core funding no. P3-0083), and the European Union's Seventh
Framework Program for research, technological development, and demonstration under the CoheaHr project (grant agreement no. HEALTH-F3-2013-603019). The funders had no role in study design, data collection and analysis, decision to publish, or preparation of the manuscript.

\section{References}

1. Bouvard V, Baan R, Straif K, Grosse Y, Secretan B, El Ghissassi F, et al; WHO International Agency for Research on Cancer Monograph Working Group. A review of human carcinogens-part B: biological agents. Lancet Oncol. 2009;10:321-2.

2. Forman D, de Martel C, Lacey CJ, Soerjomataram I, Lortet-Tieulent J, Bruni L, et al. Global burden of human papillomavirus and related diseases. Vaccine. 2012; 30:F12-23.

3. Cubie HA. Diseases associated with human papillomavirus infection. Virology. 2013;445:21-34.

4. de Martel C, Plummer M, Vignat J, Franceschi S. Worldwide burden of cancer attributable to HPV by site, country and HPV type. Int J Cancer. 2017;141:664-70.

5. Quint KD, Genders RE, de Koning MN, Borgogna C, Gariglio M, Bouwes Bavinck JN, et al. Human beta-papillomavirus infection and keratinocyte carcinomas. J Pathol. 2015;235:342-54.

6. Ferlay J, Soerjomataram I, Dikshit R, Eser S, Mathers C, Rebelo M, et al. Cancer incidence and mortality worldwide: sources, methods and major patterns in GLOBOCAN 2012. Int J Cancer. 2015;136:E359-86.

7. Poljak M, Kocjan BJ, Hošnjak L. Role of human papillomaviruses in esophageal carcinoma: an updated systematic review from 1982 to 2013. Future Virol. 2014;9:69-86.

8. Global Burden of Disease Cancer Collaboration; Fitzmaurice C, Allen C, Barber RM, Barregard L, Bhutta ZA, Brenner H, et al. Global, Regional, and National Cancer Incidence, Mortality, Years of Life Lost, Years Lived With Disability, and Disability-Adjusted Life-years for 32 Cancer Groups, 1990 to 2015: A Systematic Analysis for the Global Burden of Disease Study. JAMA Oncol. 2017;3:524-48.

9. Liyanage SS, Rahman B, Ridda I, Newall AT, Tabrizi SN, Garland SM, et al. The aetiological role of human papillomavirus in oesophageal squamous cell carcinoma: a meta-analysis. PLoS One. 2013;8:e69238.

10. Abnet CC, Arnold M, Wei WQ. Epidemiology of esophageal squamous cell carcinoma. Gastroenterology. 2017;23:7495-652.

11. Pennathur A, Gibson MK, Jobe BA, Luketich JD. Oesophageal carcinoma. Lancet. 2013;381:400-12.

12. Lagergren J, Smyth E, Cunningham D, Lagergren P. Oesophageal cancer. Lancet. 2017;390:2383-96.

13. Arnold M, Soerjomataram I, Ferlay J, Forman D. Global incidence of oesophageal cancer by histological subtype in 2012. Gut. 2015;64:381-7.

14. Arnold M, Laversanne M, Brown LM, Devesa SS, Bray F. Predicting the future burden of esophageal cancer by histological subtype: international trends in incidence up to 2030. Am J Gastroenterol. 2017;112:1247-55.

15. Cancer Genome Atlas Research Network; Kim J, Bowlby R, Mungall AJ, Robertson AG, Odze RD, Cherniack AD, et al. Integrated genomic characterization of oesophageal carcinoma. Nature. 2017;541:169-75.

16. Wu C, Wang Z, Song X, Feng XS, Abnet CC, He J, et al. Joint analysis of three genome-wide association studies of esophageal squamous cell carcinoma in Chinese populations. Nat Genet. 2014;46:1001-6.

17. Syrjänen KJ. Histological changes identical to those of condylomatous lesions found in esophageal squamous cell carcinomas. Arch Geschwulstforsch. 1982; 52:283-92.

18. Poljak M, Cerar A, Seme K. Human papillomavirus infection in esophageal carcinomas: a study of 121 lesions using multiple broad-spectrum polymerase chain reactions and literature review. Hum Pathol. 1998;29:266-71.

19. Syriänen K. HPV infections and oesophageal cancer. J Clin Pathol. 2002;55:7218.

20. Syrjänen K. HPV and oesophageal carcinoma. In: Campo MS, editor. Papillomavirus research: from natural history to vaccines and beyond. Poole, UK: Caister Academic Press; 2006. p. 229-53.

21. Syriänen K. Esophageal squamous cell carcinoma: epidemiology and risk fac tors. In: Human papillomavirus (HPV) involvement in esophageal carcinogenesis. New York: Nova Science Publishers Inc.; 2010. p. 29-38.

22. Syriänen K. Geographic origin is a significant determinant of human papillomavirus prevalence in oesophageal squamous cell carcinoma: systematic review and meta-analysis. Scand J Infect Dis. 2013;45:1-18.

23. Liu H, Li J, Diao M, Cai Z, Yang J, Zeng Y. Statistical analysis of human papillomavirus in a subset of upper aerodigestive tract tumors. J Med Virol. 2013;85:177585 .

24. Hardefeldt HA, Cox MR, Eslick GD. Association between human papillomavirus (HPV) and oesophageal squamous cell carcinoma: a meta-analysis. Epidemiol Infect. 2014;142:1119-37.
25. Li X, Gao C, Yang Y, Zhou F, Li M, Jin Q, et al. Systematic review with meta-analysis: the association between human papillomavirus infection and oesophageal cancer. Aliment Pharmacol Ther. 2014;39:270-81.

26. Yong F, Xudong N, Lijie T. Human papillomavirus types 16 and 18 in esophagus squamous cell carcinoma: a meta-analysis. Ann Epidemiol. 2013;23:726-34.

27. Zhang SK, Guo LW, Chen Q, Zhang M, Liu SZ, Quan PL, et al. Prevalence of human papillomavirus 16 in esophageal cancer among the Chinese population: a systematic review and meta-analysis. Asian Pac J Cancer Prev. 2014;15:10143-9.

28. Zhang SK, Guo LW, Chen Q, Zhang M, Liu SZ, Quan PL, et al. The association between human papillomavirus 16 and esophageal cancer in Chinese population: a meta-analysis. BMC Cancer. 2015;15:1096.

29. Guo LW, Zhang SK, Liu SZ, Chen Q, Zhang M, Quan PL, et al. Human papillomavirus type-18 prevalence in oesophageal cancer in the Chinese population: a meta-analysis. Epidemiol Infect. 2016;144:469-77.

30. Michaelsen SH, Larsen CG, von Buchwald C. Human papillomavirus shows highly variable prevalence in esophageal squamous cell carcinoma and no significant correlation to p16INK4a overexpression: a systematic review. J Thorac Oncol. 2014;9:865-71.

31. Wang L, Li J, Hou J, Li M, Cui X, Li S, et al. p53 expression but not p16INK4A correlates with human papillomavirus-associated esophageal squamous cell carcinoma in Kazakh population. Infect Agent Cancer. 2016;11:19.

32. Halec G, Schmitt M, Egger S, Abnet CC, Babb C, Dawsey SM, et al. Mucosal alpha-papillomaviruses are not associated with esophageal squamous cell carcinomas: lack of mechanistic evidence from South Africa, China and Iran and from a world-wide meta-analysis. Int J Cancer. 2016;139:85-98.

33. Petrick JL, Wyss AB, Butler AM, Cummings C, Sun X, Poole C, et al. Prevalence of human papillomavirus among oesophageal squamous cell carcinoma cases: systematic review and meta-analysis. Br J Cancer. 2014;110:2369-77.

34. Liyanage SS, Segelov E, Garland SM, Tabrizi SN, Seale H, Crowe PJ, et al. Role of human papillomaviruses in esophageal squamous cell carcinoma. Asia Pac Clin Oncol. 2013;9:12-28.

35. Wang J, Zhao L, Yan H, Che J, Huihui L, Jun W, et al. A Meta-Analysis and System atic Review on the Association between Human Papillomavirus (Types 16 and 18) Infection and Esophageal Cancer Worldwide. PLoS One. 2016;11:e0159140.

36. Guo F, Liu Y, Wang X, He Z, Weiss NS, Madeleine MM, et al. Human papillomavirus infection and esophageal squamous cell carcinoma: a case-control study. Cancer Epidemiol Biomarkers Prev. 2012;21:780-5.

37. Hu JM, Li L, Chen YZ, Pang LJ, Yang L, Liu CX, et al. Human papillomavirus type 16 infection may be involved in esophageal squamous cell carcinoma carcinogenesis in Chinese Kazakh patients. Dis Esophagus. 2013;26:703-7.

38. Liu T, Liu Q, Liang M, Zheng S, Li XL, Lu X, et al. Viral load of HPV 16/18 in esophageal squamous cell carcinoma in three ethnic groups living in Xinjiang Autonomous Region, China. Mol Biol Rep. 2013;40:2045-52.

39. Wang YF, Wang XS, Gao SG, Chen Q, Yang YT, Xiao ZY, et al. Clinical significance of combined detection of human papilloma virus infection and human telomerase RNA component gene amplification in patients with squamous cell carcinoma of the esophagus in northern China. Eur J Med Res. 2013;18:11.

40. Cao F, Han H, Zhang F, Wang B, Ma W, Wang Y, et al. HPV infection in esophageal squamous cell carcinoma and its relationship to the prognosis of patients in northern China. Scientific World J. 2014;2014:804738.

41. Chen WG, Yang CM, Xu LH, Zhang N, Liu XY, Ma YG, et al. Gene chip technology used in the detection of HPV infection in esophageal cancer of Kazakh Chinese in Xinjiang Province. J Huazhong Univ Sci Technolog Med Sci. 2014;34:343-7.

42. Cui X, Chen Y, Liu L, Li L, Hu J, Yang L, et al. Heterozygote of PLCE1 rs2274223 increases susceptibility to human papillomavirus infection in patients with esoph ageal carcinoma among the Kazakh populations. I Med Virol. 2014:86:608-17.

43. Liu HY, Zhou SL, Ku JW, Zhang DY, Li B, Han XN, et al. Prevalence of human papillomavirus infection in esophageal and cervical cancers in the high incidence area for the two diseases from 2007 to 2009 in Linzhou of Henan Province, Northern China. Arch Virol. 2014;159:1393-401.

44. Teng H, Li X, Liu X, Wu J, Zhang J. The absence of human papillomavirus in esophageal squamous cell carcinoma in East China. Int J Clin Exp Pathol. 2014; 7:4184-93

45. Dong HC, Cui XB, Wang LH, Li M, Shen YY, Zhu JB, et al. Type-specific detection of human papillomaviruses in Kazakh esophageal squamous cell carcinoma by genotyping both E6 and L1 genes with MALDI-TOF mass spectrometry. Int J Clin Exp Pathol. 2015;8:13156-65. 
46. Mehryar MM, Li SY, Liu HW, Li F, Zhang F, Zhou YB, et al. Prevalence of human papillomavirus in esophageal carcinoma in Tangshan, China. World J Gastroenterol. 2015;21:2905-11.

47. Wang WL, Wang YC, Lee CT, Chang CY, Lo JL, Kuo YH, et al. The impact of human papillomavirus infection on the survival and treatment response of patients with esophageal cancers. J Dig Dis. 2015;16:256-63.

48. Xi R, Zhang X, Chen X, Pan S, Hui B, Zhang L, et al. Human papillomavirus 16 infection predicts poor outcome in patients with esophageal squamous cell carcinoma. Onco Targets Ther. 2015;8:573-81.

49. Zou N, Yang L, Chen L, Li T, Jin T, Peng H, et al. Heterozygote of TAP1 Codon637 decreases susceptibility to HPV infection but increases susceptibility to esophageal cancer among the Kazakh populations. J Exp Clin Cancer Res. 2015:34:70.

50. Wang WL, Wang YC, Chang CY, Lo JL, Kuo YH, Hwang TZ. Human papillomavirus infection on initiating synchronous esophageal neoplasia in patients with head and neck cancer. Laryngoscope. 2016;126:1097-102.

51. Zhang D, Zhang W, Liu W, Mao Y, Fu Z, Liu J, et al. Human papillomavirus infection increases the chemoradiation response of esophageal squamous cell carcinoma based on P53 mutation. Radiother Oncol. 2017;124:155-60.

52. Li S, Shen H, Liu Z, Li N, Yang S, Zhang K, et al. Integration of human papillomavirus 16 in esophageal carcinoma samples. Infect Agent Cancer. 2017;12:53.

53. Schäfer G, Kabanda S, van Rooyen B, Marušič MB, Banks L, Parker MI. The role of inflammation in HPV infection of the oesophagus. BMC Cancer. 2013;13:185.

54. Kayamba V, Bateman AC, Asombang AW, Shibemba A, Zyambo K, Banda T, et al. HIV infection and domestic smoke exposure, but not human papillomavirus, are risk factors for esophageal squamous cell carcinoma in Zambia: a case-control study. Cancer Med. 2015;4:588-95.

55. Gupta N, Barwad A, Rajwanshi A, Kochhar R. Prevalence of human papillomavirus in esophageal carcinomas: a polymerase chain reaction-based study. Acta Cytol. 2012;56:80-4.

56. Pandilla R, Kotapalli V, Gowrishankar S, Chigurupati M, Patnaik S, Uppin S, et al. Distinct genetic aberrations in oesophageal adeno and squamous carcinoma. Eur J Clin Invest. 2013;43:1233-9.

57. Vaiphei K, Kochhar R, Bhardawaj S, Dutta U, Singh K. High prevalence of human papillomavirus in esophageal squamous cell carcinoma: a study in paired samples. Dis Esophagus. 2013;26:282-7.

58. Abdirad A, Eram N, Behzadi AH, Koriyama C, Parvaneh N, Akiba S, et al. Human papillomavirus detected in esophageal squamous cell carcinoma in Iran. Eur J Intern Med. 2012;23:59-62.

59. Noori S, Monabati A, Ghaderi A. The prevalence of human papilloma virus in esophageal squamous cell carcinoma. Iran J Med Sci. 2012;37:126-33.

6o. Yahyapour Y, Shamsi-Shahrabadi M, Mahmoudi M, Siadati S, Shahryar SS, Shokri-Shirvani J, et al. Evaluation of human papilloma virus infection in patients with esophageal squamous cell carcinoma from the Caspian Sea area, north of Iran. Asian Pac J Cancer Prev. 2012;13:1261-6.

61. Haeri H, Mardany O, Asadi-Amoli F, Shahsiah R. Human papilloma virus and esophageal squamous cell carcinoma. Acta Med Iran. 2013;51:242-5.

62. Soheili F, Heidary N, Rahbar M, Nikkho B, Fotouhi O, Afkhamzadeh A, et al. Hu man papillomavirus and its clinical relevance in oesophageal squamous cell carcinoma in a Kurdish population in the west of Iran. Infect Dis (Lond). 2016;48: 270-73.

63. Yahyapour Y, Sadeghi F, Alizadeh A, Rajabnia R, Siadati S. Detection of Merkel cell polyomavirus and human papillomavirus in esophageal squamous cell carcinomas and non-cancerous esophageal samples in northern Iran. Pathol Oncol Res. 2016;22:667-72.

64. de Oliveira Mota MT, Bonilha JL, Rosa BM, de Arruda JG, Soares FA, Vassallo J, et al. High-risk human papillomaviruses in two different primary tumors in the same patient. Int J Urol. 2013;20:1046-8.

65. Herbster S, Ferraro CT, Koff NK, Rossini A, Kruel CD, Andreollo NA, et al. HPV infection in Brazilian patients with esophageal squamous cell carcinoma: interpopulational differences, lack of correlation with surrogate markers and clinicopathological parameters. Cancer Lett. 2012;326:52-8.

66. Antunes LC, Prolla JC, de Barros Lopes A, da Rocha MP, Fagundes RB. No evidence of HPV DNA in esophageal squamous cell carcinoma in a population of southern Brazil. World J Gastroenterol. 2013;19:6598-603.

67. da Costa AM, Fregnani JHTG, Pastrez PRA, Mariano VS, Neto CS, Guimarães DP, et al. Prevalence of high risk HPV DNA in esophagus is high in Brazil but not related to esophageal squamous cell carcinoma. Histol Histopathol. 2017:11929.

68. Liyanage SS, Segelov E, Malik A, Garland SM, Tabrizi SN, Cummins E, et al. A case-control study of the role of human papillomavirus in oesophageal squamous cell carcinoma in Australia. J Oncol. 2014;2014:236482.
69. Georgantis G, Syrakos T, Agorastos T, Miliaras S, Gagalis A, Tsoulfas G, et al. Detection of human papillomavirus DNA in esophageal carcinoma in Greece. World J Gastroenterol. 2015;21:2352-7.

70. Dąbrowski A, Kwaśniewski W, Skoczylas T, Bednarek W, Kuźma D, GoździckaJózefiak A. Incidence of human papilloma virus in esophageal squamous cell carcinoma in patients from the Lublin region. World J Gastroenterol. 2012;18:573944 .

71. Löfdahl HE, Du J, Näsman A, Andersson E, Rubio CA, Lu Y, et al. Prevalence of human papillomavirus (HPV) in oesophageal squamous cell carcinoma in relation to anatomical site of the tumour. PLoS ONE. 2012;7:e46538.

72. Türkay DÖ, Vural Ç, Sayan M, Gürbüz Y. Detection of human papillomavirus in esophageal and gastroesophageal junction tumors: A retrospective study by real-time polymerase chain reaction in an instutional experience from Turkey and review of literature. Pathol Res Pract. 2016;212:77-82.

73. Landau M, Goldblum JR, Deroche T, Dumot J, Downs-Kelly E, Rice TW, et al. Esophageal carcinoma cuniculatum: report of 9 cases. Am J Surg Pathol. 2012;36:8-17.

74. Doxtader EE, Katzenstein AL. The relationship between p 16 expression and highrisk human papillomavirus infection in squamous cell carcinomas from sites other than uterine cervix: a study of 137 cases. Hum Pathol. 2012;43:327-32.

75. Ludmir EB, Palta M, Zhang X, Wu Y, Willett CG, Czito BG. Incidence and prognostic impact of high-risk HPV tumor infection in cervical esophageal carcinoma. J Gastrointest Oncol. 2014;5:401-7.

76. Xi R, Pan S, Chen X, Hui B, Zhang L, Fu S, et al. HPV16 E6-E7 induces cancer stem-like cells phenotypes in esophageal squamous cell carcinoma through the activation of $\mathrm{Pl}_{3} \mathrm{~K} /$ Akt signaling pathway in vitro and in vivo. Oncotarget. 2016;7:57050-65.

77. Rajendra S, Robertson IK. Similar immunogenetics of Barrett's oesophagus and cervical neoplasia: is HPV the common denominator? J Clin Pathol. 2010;63:1-3.

78. Baghdadi J, Chaudhary N, Pei Z, Yang L. Microbiome, innate immunity, and esophageal adenocarcinoma. Clin Lab Med. 2014;34:721-32.

79. Kamath AM, Wu TT, Heitmiller R, Daniel R, Shah KV. Investigation of the association of esophageal carcinoma with human papillomaviruses. Dis Esophagus. 2000;13:122-4.

8o. Tornesello ML, Monaco R, Nappi O, Buonaguro L, Buonaguro FM. Detection of mucosal and cutaneous human papillomaviruses in oesophagitis, squamous cell carcinoma and adenocarcinoma of the oesophagus. J Clin Virol. 2009;45:28-33.

81. Wang X, Tian X, Liu F, Zhao Y, Sun M, Chen D, et al. Detection of HPV DNA in esophageal cancer specimens from different regions and ethnic groups: a descriptive study. BMC Cancer. 2010;10:19.

82. Iyer A, Rajendran V, Adamson CS, Peng Z, Cooper K, et al. Human papillomavirus is detectable in Barrett's esophagus and esophageal carcinoma but is unlikely to be of any etiologic significance. J Clin Virol. 2011;50:205-8.

83. Feng S, Zheng J, Du X, Tan Y, Yang H, Zhang H, et al. Human papillomavirus was not detected by PCR using multiple consensus primer sets in esophageal adenocarcinomas in Chinese patients. J Med Virol. 2013;85:1053-7.

84. Antonsson A, Knight L, Whiteman DC; Australian Cancer Study. Human papillomavirus not detected in esophageal adenocarcinoma tumor specimens. Cancer Epidemiol. 2016;41:96-8.

85. Rajendra S, Wang B, Snow ET, Sharma P, Pavey D, Merrett N, et al. Transcriptionally active human papillomavirus is strongly associated with Barrett's dysplasia and esophageal adenocarcinoma. Am J Gastroenterol. 2013;108:1082-93.

86. Rajendra S, Wang B, Merrett N, Sharma P, Humphris J, Lee HC, et al. Genomic analysis of HPV-positive versus HPV-negative oesophageal adenocarcinoma identifies a differential mutational landscape. J Med Genet. 2016;53:227-31.

87. Stransky N, Egloff AM, Tward AD, Kostic AD, Cibulskis K, Sivachenko A, et al. The mutational landscape of head and neck squamous cell carcinoma. Science. 2011;333:1157-60.

88. Mirabello L, Yeager M, Yu K, Clifford GM, Xiao Y, Zhu B, et al. HPV16 E7 Genetic Conservation Is Critical to Carcinogenesis. Cell. 2017;170:1164-74.

89. Rajendra S, Wang B, Pavey D, Sharma P, Yang T, Lee CS, et al. Persistence of human papillomavirus, overexpression of p53, and outcomes of patients after endoscopic ablation of Barrett's esophagus. Clin Gastroenterol Hepatol. 2015;13: 1364-8.e5.

90. Rajendra S, Yang T, Xuan W, Sharma P, Pavey D, Lee CS, et al. Active human papillomavirus involvement in Barrett's dysplasia and oesophageal adenocarcinoma is characterized by wild-type $\mathrm{p} 53$ and aberrations of the retinoblastoma protein pathway. Int J Cancer. 2017;141:2037-49.

91. Poljak M, Seme K, Gale N. Detection of human papillomaviruses in tissue specimens. Adv Anatomic Pathol. 1998;5:216-34. 